\title{
Simulation and Experimental Analysis of the Vibration System Composed of HEM and Test Bench
}

\author{
Jingjing Yao ${ }^{a}$, Zhijun Chen ${ }^{b}$ \\ Chongqing College of Electronic Engineering, Chongqing 401331, China \\ ay119jj@126.com, bchenzhijun_2@163.com
}

Keywords: hydraulic mounting, test bench, simulation, experimental.

\begin{abstract}
According to the structure of hydraulic mounting and working principle of the electro-hydraulic servo test bench, established the system model constituted by HEM and test bench, then did the simulation analysis and experimental verification for the vibration system, the results show that the model is the correctness and reliability.
\end{abstract}

\section{Introduction}

Vibration is one of the important factors which affect the performance of vehicle, and the powertrain vibration is one of the main exciting source of vehicle vibration. So powertrain vibration isolation system is a key technology to reduce the engine vibration and noise. Powertrain mounting device is a powertrain vibration isolation component, which can reduce the vibration transmission so that makes the vehicle vibration and noise control were improved. Studying powertrain device is an effective approach to improve the performance of vehicle vibration isolation. Automobile shock absorber electro-hydraulic servo test bench can simulate all kinds of vibration of the car, research the performance of hydraulic mounting by using the test bench can provide a powerful basis for optimal design of powertrain mounting devices.

\section{The structure and mathematical model of hydraulic mounting}

Reasonable powertrain mount system can reduce the vibration transmission. HEM( Hydraulic engine mount) is widely used in the mounting system to isolate vibration, the advantage of the HEM is compact structure, light quality, and better performance. Hydraulic mounting system is widely used in vibration isolation components presently. Figure 1 is the structure chart of hydraulic mounting developed by our team members. Rubber main spring 3 is the main bearing of hydraulic mount components, rubber spring and basement membrane constitutes the body part of hydraulic shock absorber, inertia channel divide body cavity into two liquid chamber----upper chamber and bottom chamber, which are filled with liquid. HEM is equivalent to parallel a hydraulic shock absorber based on the rubber mount. HEM is designed up and down with inertia channel in the rubber mount, the liquid flows between in the chambers that can consume vibration energy because of liquid damping, so as to achieve the purpose of vibration isolation.

In order to study the performance of the HEM, the model of HEM is established. When establishing the model, we use the following assumptions to make the model can highlight the main influencing factors of the HEM.

The liquid is incompressible.

Because the influence of the dynamic characteristics cause by HEM on vertical direction is larger than lateral direction, so only a mathematical model of HEM on $\mathrm{Z}$ direction is established in this paper.

The fluid leakage of the upper and bottom chamber is ignored.

The inertia pressure loss caused by the movement of the fluid is ignored.

The liquid density changing with pressure, temperature is very small, usually neglected.

The liquid resistance coefficient is constant in the inertia channel. 


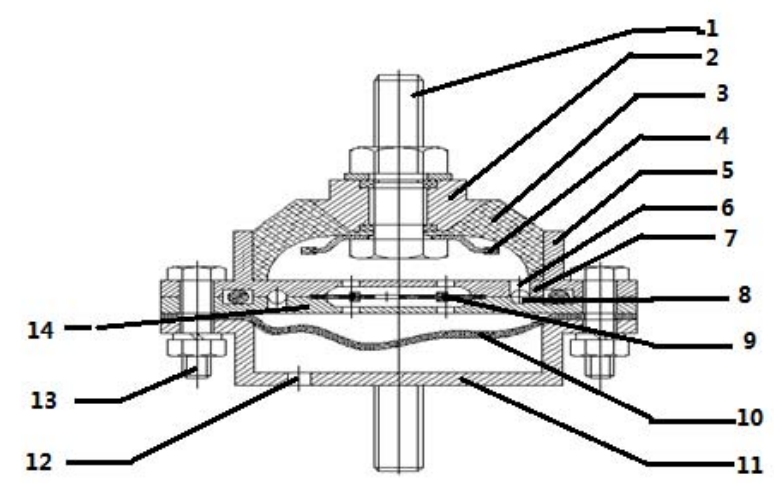

Coupling bolt 2. Metal skeleton 3. Rubber main spring 4. Limit support

5. Metal cover 6. Entrance of inertia channel 7. The upper part of inertia channel

8. Inertia channel 9. Decoupling plate 10. The basement membrane of bottom chamber 11. Pedestal

12. Venthole 13. Coupling bolt 14. The bottom part of inertia channel

Fig. 1 The structure chart of HEM

Based on the above assumptions, the simplified model of hydraulic mount system is shown in figure 2:

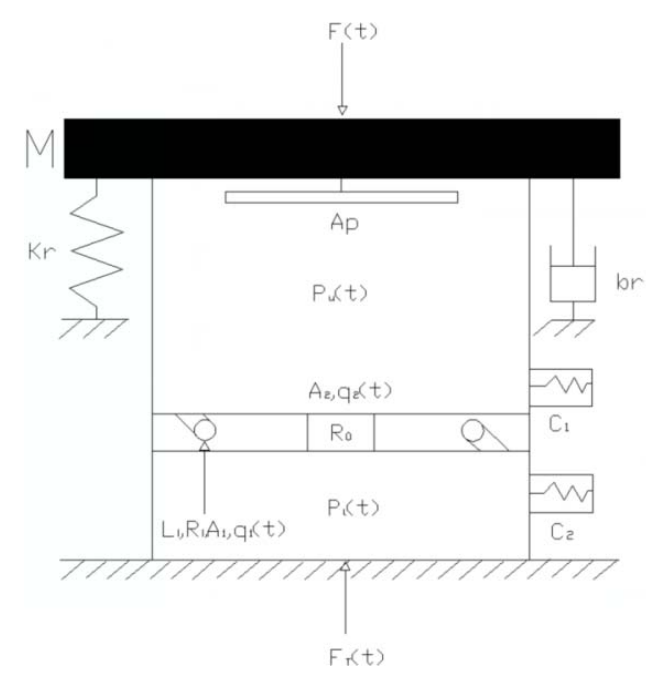

Fig.2 The hydraulic model of HEM

The symbolic meaning is as follows: $K_{r}$ is the stiffness of the main rubber spring; $b_{r}$ is the equivalent damping coefficient of the rubber main spring; $A_{p}$ is the equivalent piston area; $M$ is the powertrain quality that beared by EHM. $F(t)$ is the input force by HEM; $F_{T}(t)$ is the force passed to frame from HEM; $q_{1}(t)$ is the fluid flowing from upper chamber flow into bottom chamber by inertia channel; $q_{2}(t)$ is the fluid flowing from upper chamber flow into bottom chamber by orifice; $P_{u}(t)$ is the pressure of the upper chamber; $P_{l}(t)$ is the pressure of the bottom chamber; $C_{1}$ is of the volume flexibility of upper chamber; $C_{2}$ is of the volume flexibility of bottom chamber, the value of $C_{1}, C_{2}$ are the reciprocal of volume stiffness $K_{1}, K_{2} ; I_{i}$ is the fluid inertia effect of flowing fluid in inertia channel, $R_{i}$ is the fluid resistance effect of flowing fluid in inertia channel, namely the fluid inertia coefficient and resistance coefficient; $A_{i}$ and $R_{0}$ is the cross-sectional area of inertia channel and orifice respectively.

When establishing the mathematical model, use vibration differential equations for the mechanical part and volume and pressure changing equations for fluid part.

The fluid continuity equation as follow:

$$
A_{p} \dot{x}=A_{i} \dot{x}_{i}+K_{1}\left(p_{u}-p_{l}\right)
$$


In the formula: $A_{p}$ is the equivalent piston area of the upper chamber; $x$ is the displacement of the powertrain; $p_{u}$ is the pressure of the upper chamber; $p_{l}$ is the pressure of the bottom chamber; $A_{i}$ is the cross-sectional area of the inertia channel; $x_{i}$ is the fluid displacement of the inertia channel

Take the liquid in inertia channel as the research object, establish a force balance equation:

$m_{i} \ddot{x}_{i}+R_{i} \dot{x}_{i}=A_{i}\left(p_{u}-p_{l}\right)$

In the formula: $m_{i}$ is the liquid quality in inertia channel; $R_{i}$ is the resistance coefficient of the liquid flowing in inertia channel.

Take the whole systems as the research object, establish the following two balance equations:

$F_{T}=K_{r} x+b_{r} \dot{x}+A_{p}\left(p_{u}-p_{l}\right)$

$M \ddot{x}=F-F_{T}$

In the formula: $F$ is the excitation force of engineer; $M$ is the engineer quality.

\section{The work principle and mathematical model of electro-hydraulic servo test bench}

The working principle of the electro-hydraulic servo test bench is shown in figure 3 . Microcomputer gives orders according to the test requirements, PCI2306 - DA card converts digital signals into analog signals, then the input device produces electrical signals, which are amplified and regulated by the servo amplifier; The enlarged electrical signals control the hydraulic power components through the electro-hydraulic servo valve, so the hydraulic cylinder piston can move according to the requirement of the direction and speed, then drive the tested components. In order to realize accurate control of test bench, the feedback sensor is joined in the control system. When making bench test, the sensor detects the output displacement signal of the hydraulic cylinder at any time, feedback in the input after conversion into electrical signal, and comparing with the input signal, only when the approximation error of the input signal and feedback signal is similar to zero, the tested components produce the expected movement.

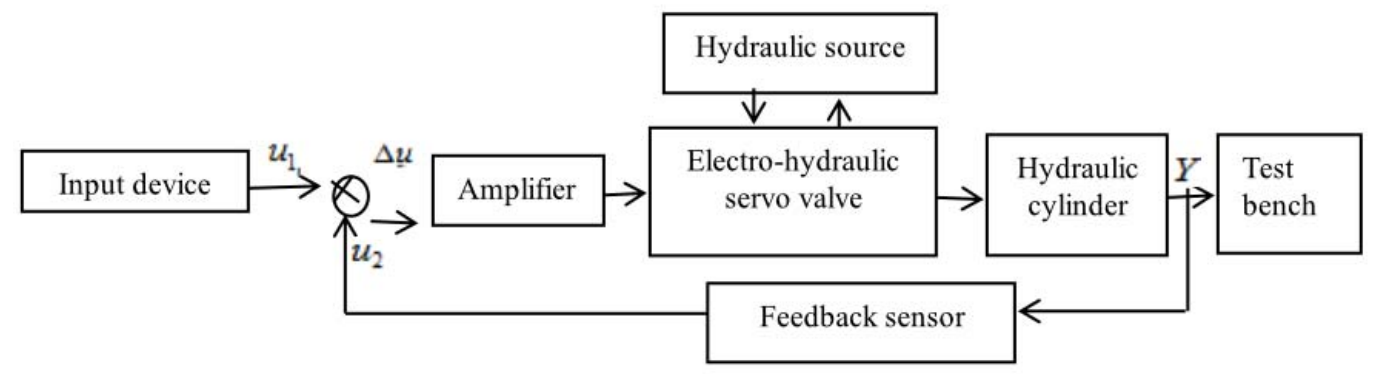

Fig.3 The working principle graph of the electro-hydraulic servo test bench

\section{The system model and simulation of HEM — vibration test bench}

According to the formula (1), (2) and (3), used Matlab/Simulink simulation tool to establish the simulation diagram of the HEM. Then simulate the model, get the linear model of system, the frequency characteristics of the HEM is shown in figure 4.

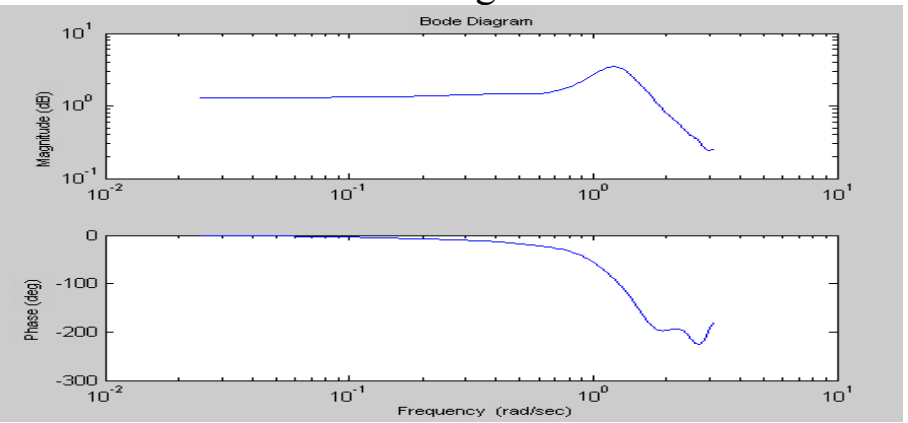

Fig.4 The frequency characteristics of the HEM model 
According to the formula (4), (5) and (6), established a simulation model of hydraulic power components.

Basing on each link transfer function of HEM and test bench determined above, according to the transitive relation of each link, established the simulation model of the vibration system consist by HEM and electro-hydraulic servo test bench. The model is shown in figure 5.

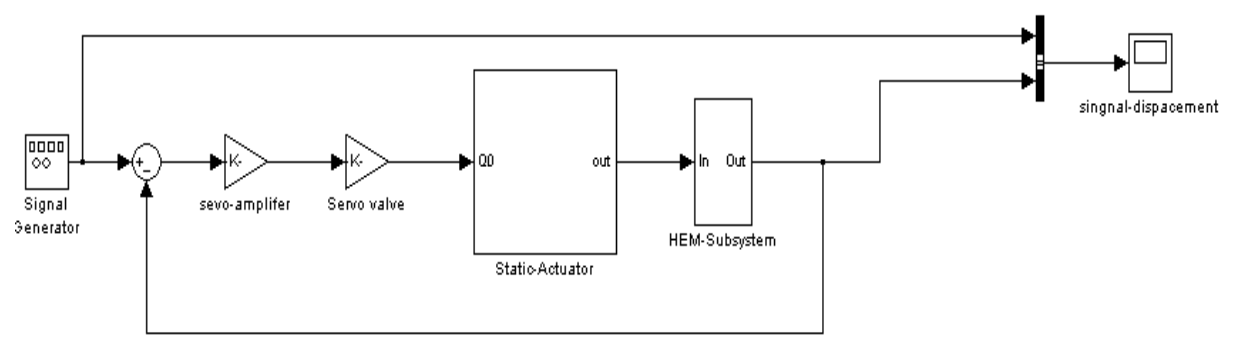

Fig.5 The simulation model of the vibration system

Figure 6 is the comparison curve of input and output when the input is sinusoidal signal that amplitude is 1 and the frequency is $5 \mathrm{~Hz}$. Figure 7 is the displacement response curve when simulate the road input.

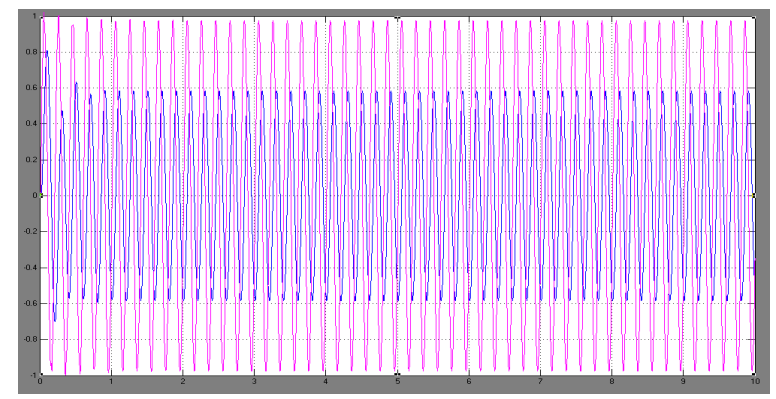

Fig.6 The comparison curve of input sinusoidal signal frequency is $5 \mathrm{~Hz}$

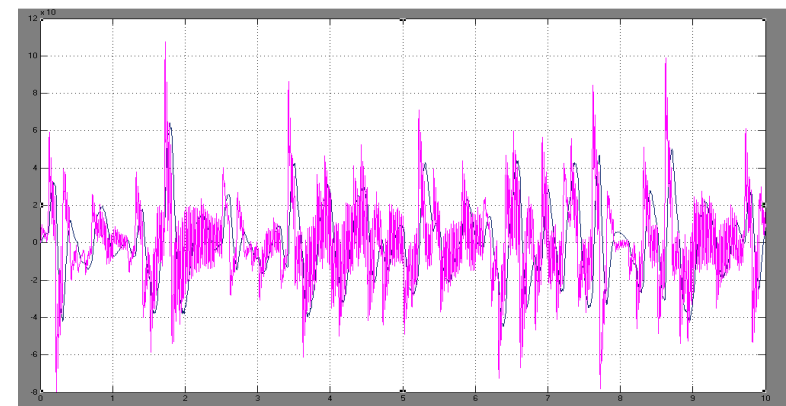

Fig.7 The displacement response curve when simulate the road input

It can be seen from the graph that the test bench can move follow the given input signal instruction. The HEM has good attenuation effects for the vibration signal, especially played a very good role on isolation in high frequency.

\section{The test data analysis}

To verify the validity of the established model, we used the electro-hydraulic servo test bench for testing a processing HEM to test its performance. When testing, the excitation signal of test bench is the simulation input of the powertrain vibration. The output signals are displacement and force signal that attenuated by HEM. According to the requirement of test and the characteristics of the test system, we used the sine signal as the test excitation signal, whose amplitude can adjust according to the frequency.

A large amount of data is obtained by the test, according to those data we got the frequency response potter curve of the HEM system, as shown in figure 9. 


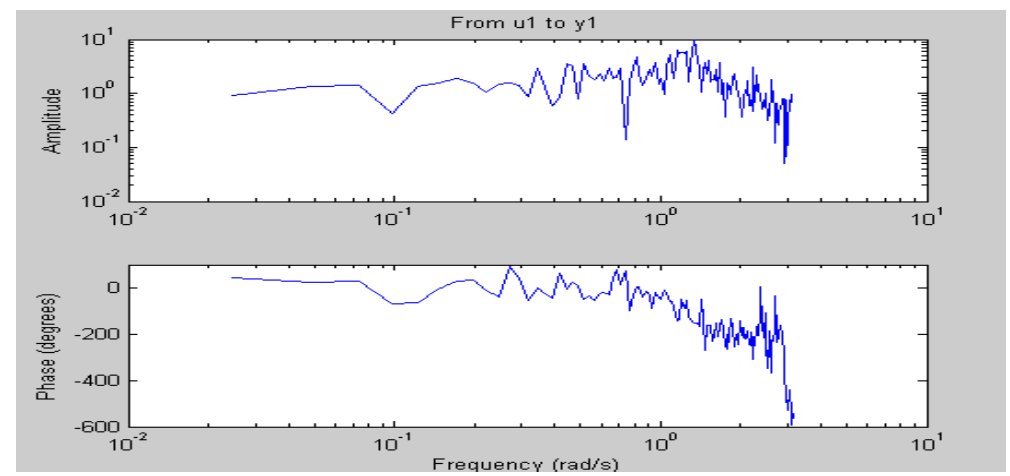

Fig.9 The frequency response potter curve

Comparing the figure 5 and figure 9 about frequency characteristic curve of the HEM, it can be seen that the trend between the frequency response curve of simulation model and the frequency response curve get from the test data is consistent, that can confirm the reliability of the model.

\section{Conclusion}

In this paper, according to the principle of the HEM, the mathematical model of the HEM has been established by using the theory of mechanical and fluid. The system corresponding motion equation has been derived according to the transfer characteristics of the hydraulic system and the model of the electro-hydraulic servo test bench has been determined. The model of the vibration system composed of HEM and test bench has been established by using MATLAB/Simulink simulation tool, and then the established model has been analyzed through simulation. After that we tested the HEM components, analyzed and processed the test data, then found that the system frequency characteristics are consistent with the simulation curve. Thus the mathematical model and simulation model is proved to be correct. The study of the HEM in this paper will play an important part to improve the vibration and noise of engine.

\section{References}

[1] Y.H. Yu. Review of Vehicle Engine Mounting. The International Journal of Global Energy Issues. (2000) No.4, p.42.

[2] Y. Guo, CH. Chen, and W. Y. Wang, et al. Emulation and Analysis of Damper Test Bench. Journal of Xihua University.Vol.26 (2007) No.1, p.22-24.

[3] H.T. Min, Y.Lin and Y.L. Xiong et al. Study on Dynamic Characteristic of Light Passenger Car's Hydraulic Mounting. Journal of Jilin University. Vol. 33 (2003) No.2, p.35.

[4] Z.H. Lv, J. Luo and R.L. Fan et al. Design and Analysis Method for Improving Vibration Isolation Performance of Automotive Powerplant Mounting Systems. The Chinese Mechanical Engineering.Vol. 14 (2003) No.3, p.265-270.

[5] T.L.Wang, B. Zhang and X.J. Tian et al. Electrohydraulic Servo Damper Test Bench. MachineTool and Hydraulics.(2003) No.2, p.224-226. 